\title{
\&ु \\ Critical events, entropy, and the grain boundary character distribution
}

\author{
K. Barmak* \\ Materials Research Science and Engineering Center, Department of Materials Science and Engineering, Carnegie Mellon University, \\ Pittsburgh, Pennsylvania 15213, USA \\ E. Eggeling ${ }^{\dagger}$ \\ Fraunhofer Austria Research GmbH, Visual Computing, A-8010 Graz, Austria \\ M. Emelianenko \\ Department of Mathematics, George Mason University, Fairfax, Virginia 22030, USA \\ Y. Epshteyn ${ }^{\S}$ \\ Department of Mathematics, The University of Utah, Salt Lake City, Utah 84112, USA \\ D. Kinderlehrer," R. Sharp, ${ }^{\llbracket}$ and S. Ta'asan ${ }^{* *}$ \\ Materials Research Science and Engineering Center, Center for Nonlinear Analysis and Department of Mathematical Sciences, \\ Carnegie Mellon University, Pittsburgh, Pennsylvania 15213, USA \\ (Received 6 August 2010; revised manuscript received 11 February 2011; published 18 April 2011)
}

\begin{abstract}
Mesoscale experiment and simulation permit harvesting information about both geometric features and texture in polycrystals. The grain boundary character distribution (GBCD) is an empirical distribution of the relative length [in two dimensions (2D)] or area (in 3D) of an interface with a given lattice misorientation and normal. During the growth process, an initially random distribution of boundary types reaches a steady state that is strongly correlated to the interfacial energy density. In simulation, it is found that if the given energy density depends only on lattice misorientation, then the steady-state GBCD and the energy are related by a Boltzmann distribution. This is among the simplest nonrandom distributions, corresponding to independent trials with respect to the energy. In this paper, we derive an entropy-based theory that suggests that the evolution of the GBCD satisfies a Fokker-Planck equation, an equation whose stationary state is a Boltzmann distribution. Cellular structures coarsen according to a local evolution law, curvature-driven growth, and are limited by space-filling constraints. The interaction between the evolution law and the constraints is governed primarily by the force balance at triple junctions, the natural boundary condition associated with curvature-driven growth, and determines a dissipation relation. A simplified coarsening model is introduced that is driven by the boundary conditions and reflects the network level dissipation relation of the grain growth system. It resembles an ensemble of inertia-free spring-mass dashpots. Application is made of the recent characterization of Fokker-Planck kinetics as a gradient flow for a free energy in deriving the theory. The theory predicts the results of large-scale two-dimensional simulations and is consistent with experiment.
\end{abstract}

DOI: 10.1103/PhysRevB.83.134117

PACS number(s): 61.72.Mm, 68.35.-p, 89.70.Cf, 05.70.Ln

\section{INTRODUCTION}

Cellular networks are ubiquitous in nature. They exhibit behavior on many different length and time scales and are generally metastable. Most technologically useful materials are polycrystalline microstructures composed of a myriad of small monocrystalline grains separated by grain boundaries, and thus comprise cellular networks. The energetics and connectivity of the grain boundary network plays a crucial role in determining the properties of a material across a wide range of scales. A central problem in materials is to develop technologies capable of producing an arrangement of grains that provides for a desired set of material properties. Traditionally, the focus has been on the geometric feature of size and the preferred distribution of grain orientations, termed texture. More recent mesoscale experiment and simulation permit harvesting large amounts of information about both geometric features and crystallography of the boundary network in material microstructures. ${ }^{1-5}$

A leading candidate to characterize the texture of the grain boundary population is the grain boundary character distribution. ${ }^{3}$ The grain boundary character distribution (GBCD) is an empirical distribution of the relative length [in two dimensions (2D)] or area (in 3D) of interface with a given lattice misorientation and grain boundary normal. During the growth process, an initially random grain boundary texture reaches a steady state that is strongly correlated to the interfacial energy density. In simulation, a GBCD is always found. In view of the previous work ${ }^{3,4}$ and the theory developed in this paper, it is the GBCD that should serve as a reference distribution for texture in preference to other distributions.

If the given energy depends only on lattice misorientation, then the steady-state GBCD and the interfacial energy density are related by a Boltzmann distribution. This is among the simplest nonrandom distributions, corresponding to independent trials with respect to the density. Such a simple dependence between the character distribution and the interfacial energy offers evidence that the GBCD is a material property. Why does such a simple distribution arise from such a complex system comprised of many interacting interfaces? In this paper, we attempt to answer this question. 
We outline an entropy-based theory that suggests that the evolving GBCD satisfies a Fokker-Planck equation. Coarsening in polycrystalline systems is a complicated process involving the details of material structures, chemistry, arrangement of grains in the configuration, and environment. In this context, we consider just two competing global features, as articulated by C. S. Smith: ${ }^{6}$ cell growth according to a local evolution law and space-filling constraints. We shall impose curvature-driven growth for the local evolution law, cf. Mullins. ${ }^{7}$ Space-filling requirements are managed by critical events, rearrangements of the network involving deletion of small contracting cells, and facets. The interaction between the evolution law and the constraints is, we shall discover, governed primarily by the balance of forces at triple junctions. This balance of forces, often referred to as the Herring condition, ${ }^{8}$ is the natural boundary condition associated with the equations of curvature-driven growth. It determines a dissipation relation for the network as a whole.

We introduce a simplified coarsening model driven by the boundary conditions that reflects the dissipation relation of the grain growth system. It resembles an ensemble of inertia-free spring-mass dashpots. ${ }^{9}$ For this simpler network, we learn how entropic or diffusive behavior at the large scale emerges from a dissipation relation at the scale of local evolution. The cornerstone is our novel implementation of the iterative scheme for the Fokker-Planck equation in terms of the system free energy and a Kantorovich-Rubinstein-Wasserstein metric, ${ }^{10}$ cf. also Ref. 11, which will be defined and explained later in the text. The network level nonequilibrium nature of the iterative scheme leaves free a temperature-like parameter. The entropy method is exploited to identify uniquely this parameter. To illustrate the idea, we include a simple application to the solution of the Fokker-Planck equation itself.

We present evidence that the theory predicts the results of large-scale two-dimensional simulations. ${ }^{12}$ Energy densities consisting of quadratic and quartic trigonometric polynomials are analyzed in detail. The discussion of the quartic-based energy density places in relief the entropic nature of the GBCD. It would take us rather far afield to discuss consistency with experiment, and we refer the reader to Ref. 3. A companion paper emphasizing the mathematical and simulation issues of the project is Ref. 13. A theory for the evolution of geometric features of microstructure is discussed in Refs. 14 and 15. Some of the results of the present work were announced in Refs. 16 and 12. Different treatments of texture development are given in Refs. 17-20.

\section{MESOSCALE THEORY}

Our point of departure is the common denominator theory for the mesoscale description of grain growth. This is curvature-driven growth, more precisely Eq. (2) below, for the motion of curves or arcs individually or in a network, which we employ for our local law of evolution. Boundary conditions must be imposed where the arcs meet. This condition is the Herring condition, (3), which is the natural boundary condition at equilibrium for (2). Since their appearance by Mullins for general or anisotropic growth ${ }^{7}$ and Herring, $, 2,21$ a large and distinguished body of work has grown regarding these equations. Most relevant to the work presented herein



FIG. 1. (Color online) An arc $\Gamma$ with normal $n$, tangent $b$, and lattice misorientation $\alpha$, illustrating lattice elements (reproduced from Ref. 16).

are Refs. 22-25. Let $\alpha$ denote the misorientation between two grains separated by an arc $\Gamma$, as noted in Fig. 1, with normal $n=(\cos \theta, \sin \theta)$, tangent direction $b$, and curvature $\kappa$. Let $\psi=\psi(\theta, \alpha)$ denote the energy density on $\Gamma$. So, representing the time-evolving arc $\Gamma$ in the $x=\left(x_{1}, x_{2}\right)$ plane by the vector-valued function $\xi(s, t)=\left(\xi_{1}(s, t), \xi_{2}(s, t)\right)$ of arc parameter $s$ and time $t$,

$$
\Gamma: x=\xi(s, t), \quad 0 \leqslant s \leqslant L, \quad t>0,
$$

with

$$
\begin{gathered}
b=\frac{\partial \xi}{\partial s} \text { (tangent), } n=R b \text { (normal), } \\
v=\frac{\partial \xi}{\partial t} \text { (velocity), } v_{n}=v \cdot n \text { (normal velocity), }
\end{gathered}
$$

where $R$ is a positive rotation of $\pi / 2$. The Mullins equation of evolution is

$$
v_{n}=\left(\psi_{\theta \theta}+\psi\right) \kappa \text { on } \Gamma .
$$

We assume that only triple junctions are stable and that the Herring condition holds at triple junctions. This means that whenever three curves $\left\{\Gamma^{(1)}, \Gamma^{(2)}, \Gamma^{(3)}\right\}$ meet at a point $p$, the force balance, (3) below, holds:

$$
\sum_{i=1, \ldots, 3}\left(\psi_{\theta} n^{(i)}+\psi b^{(i)}\right)=0 .
$$

It is easy to check $^{24}$ that the instantaneous rate of change of energy of $\Gamma$ is

$$
\frac{d}{d t} \int_{\Gamma} \psi|b| d s=-\int_{\Gamma} v_{n}^{2} d s+\left.v \cdot\left(\psi_{\theta} n+\psi b\right)\right|_{\partial \Gamma} .
$$

We turn now to a network of grains bounded by a collection of curves $\left\{\Gamma_{i}\right\}$ subject to some condition at the border of the region they occupy, such as fixed end points or periodicity, cf. Fig. 2. Our simulation is described in Refs. 26 and 27. The typical simulation consists of initializing a configuration of cells and their boundary arcs, usually by a modified Voronoi tessellation, assigning random orientations to the cells, and then solving the system (2) and (3), eliminating facets when they have negligible length and cells when they have negligible area. The simulation satisfies all known diagnostics and, in particular, when $\psi=$ const, the von Neumann-Mullins $n-6$ rule $^{28,29}$ is satisfied for each cell at each time when it is not subjected to a critical event, facet, or grain deletion.

The total energy of the system is given by

$$
E(t)=\sum_{\left\{\Gamma_{i}\right\}} \int_{\Gamma_{i}} \psi|b| d s .
$$




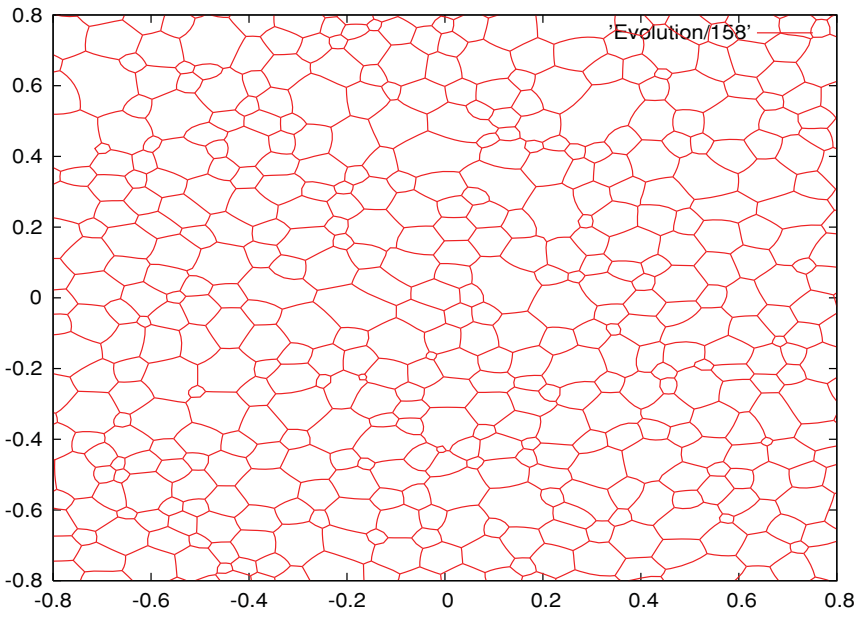

FIG. 2. (Color online) Example of an instant during the simulated evolution of a cellular network. This is part of the frame from a small simulation with constant energy density and periodic conditions at the border of the configuration.

Owing exactly to the Herring condition (3), the instantaneous rate of change of the energy is

$$
\begin{aligned}
\frac{d}{d t} E(t) & =-\sum_{\left\{\Gamma_{i}\right\}} \int_{\Gamma_{i}} v_{n}^{2} d s+\sum_{T J} v \cdot \sum\left(\psi_{\theta} n+\psi b\right) \\
& =-\sum_{\left\{\Gamma_{i}\right\}} \int_{\Gamma_{i}} v_{n}^{2} d s \\
& \leqslant 0,
\end{aligned}
$$

rendering the network dissipative for the energy in any instant absent of critical events. Indeed, in an interval $\left(t_{0}, t_{0}+\tau\right)$ where there are no critical events, we may integrate (6) to obtain a local dissipation equation,

$$
\sum_{\left\{\Gamma_{i}\right\}} \int_{t_{0}}^{t_{0}+\tau} \int_{\Gamma_{i}} v_{n}^{2} d s d t+E\left(t_{0}+\tau\right)=E\left(t_{0}\right)
$$

which bears a resemblance to the simple dissipation relation for an ensemble of inertia-free springs with friction. In the simulation, the facet interchange and cell deletion are arranged so that the inequality in (6) is maintained. In the case in which the energy density is independent of the normal direction, so $\psi=\psi(\alpha)$, the situation we will be concerned with in this paper, (2) and (3) may be expressed as

$$
\begin{gathered}
v_{n}=\psi \kappa \text { on } \Gamma \\
\sum_{i=1, \ldots, 3} \psi b^{(i)}=0 \text { at } p,
\end{gathered}
$$

where $p$ denotes a triple junction. Equation (9) is the same as the Young wetting law. ${ }^{30}$ Our interfacial energy densities $\psi$ are chosen so that

$$
1 \leqslant \psi(\alpha) \leqslant \frac{3}{2}, \quad|\alpha| \leqslant \frac{\pi}{4}
$$

(periodic with period $\pi / 2$ ) giving square symmetry that is intended to mimic cubic symmetry in three dimensions. For the range of $\psi$ in (10), one may check that (9) can always be resolved, namely, given three numbers $\psi_{i} \in[1,3 / 2]$ there are unit vectors $b_{i}$ such that

$$
\psi_{1} b_{1}+\psi_{2} b_{2}+\psi_{3} b_{3}=0 .
$$

In executing this check, one may note that if the oscillation in $\psi$ is too large, then it may not be possible to fulfill the Young law condition in general; cf. Ref. 2 for a discussion of the issue. To develop the GBCD, the collection of initial orientations must be sufficiently random, since for this type of density, all misorientations are drawn from the initial list of pairwise differences of cell orientations. ${ }^{31}$

For this situation, we define the GBCD with $\Omega=\left(-\frac{\pi}{4}, \frac{\pi}{4}\right)$,

$$
\begin{aligned}
& \rho(\alpha, t)=\text { relative length of arc of misorientation } \\
& \alpha \text { at time } t \text {, normalized so that } \\
& \qquad \int_{\Omega} \rho d \alpha=1 .
\end{aligned}
$$

\section{SIMPLIFIED COARSENING MODEL}

A significant difficulty in developing a theory for the GBCD, and understanding texture development in general, lies in the lack of understanding of the consequences of rearrangement events or critical events, facet interchange, and grain deletion on misorientations and grain size. For example, in Fig. 3, the average area of six-faceted grains during a growth experiment on an $\mathrm{Al}$ thin film $\mathrm{m}^{32}$ and the average area of six-faceted cells in a typical simulation ${ }^{33}$ both increase with time. Note that the von Neumann-Mullins rule is that the area $A_{n}$ of a cell with $n$ facets satisfies

$$
A_{n}^{\prime}(t)=c(n-6),
$$

when $\psi=$ const and triple junctions meet at angles of $2 \pi / 3$. This is thought to hold approximately when anisotropy is small. The von Neumann-Mullins rule does not fail in the example in Fig. 3, of course, but cells observed at later times had $7,8, \ldots$ facets at earlier times. The trend of increase in average area over time holds for all facet classes. Thus in the network setting, critical events and subsequent rearrangement play a major role. Although we may be reasonably confident that small cells with small numbers of facets will be deleted, their resulting effect on the configuration appears to be essentially random.

We shall study this by a simplified model that retains critical events and kinetics but neglects curvature-driven motion of the boundaries. It is an abstraction of the role of triple junctions in the presence of the rearrangement events. We have used this model to develop a statistical theory for critical events. ${ }^{34-36}$ It has been found to have its own GBCD, which we shall now study.

Our theme will be that the GBCD statistic for the simplified model resembles the solution of a Fokker-Planck equation obtained via the mass transport implicit scheme. The first part of the discussion consists in introducing this model. The simplified model is formulated as a gradient flow, which results in a dissipation inequality analogous to the one found for the coarsening grain network. Because of this simplicity, it will be possible to "upscale" the network level system description to a higher-level GBCD description that accommodates irreversibility. As this changes the ensemble, 

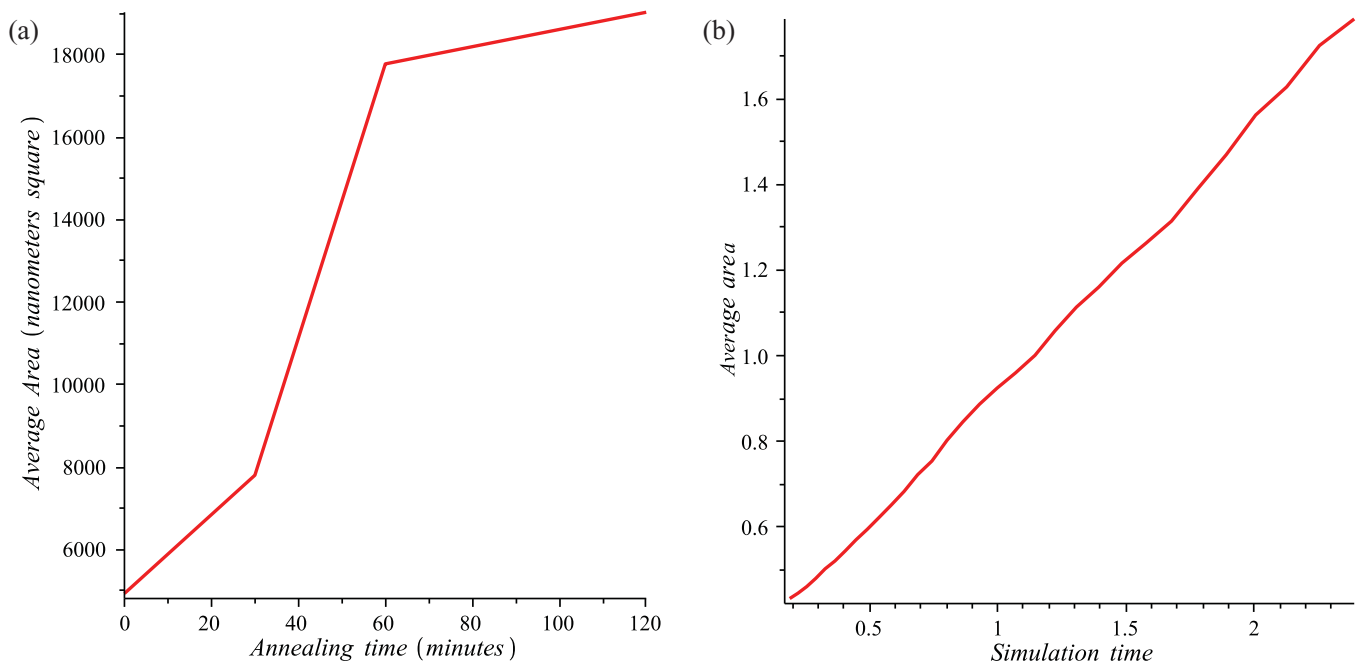

FIG. 3. (Color online) The average area of six-sided cell populations during coarsening in two different cellular systems showing that the von Neumann-Mullins $n-6$ rule (12) does not hold at the scale of the network. (a) In an experiment on Al thin film, (b) a typical simulation (arbitrary units). Please refer to the first paragraph of Sec. III for additional explanation.

following Boltzmann, there is an entropic contribution, which we take in the form of configurational entropy. A more useful dissipation inequality is obtained by modifying the "velocity" term to be a true viscous term, which now brings us to the realm of the Kantorovich-Rubinstein-Wasserstein implicit scheme, sometimes referred to as the JKO scheme. At this stage, we explain how we may appeal to the Fokker-Planck paradigm.

The second part of the discussion, in Sec. IV, will be our argument to validate this paradigm. We do not know that the statistic solves the Fokker-Planck partial differential equation (PDE), but we ask if it shares important aspects of Fokker-Planck behavior. A defining characteristic of the Fokker-Planck equation, and diffusion equations in general, is the exponential decay of their solutions to equilibrium. We give evidence for this by asking for the unique "temperature-like" parameter that minimizes the relative entropy over a long time. The empirical stationary distribution and Boltzmann distribution with the special parameter value are in excellent agreement; see Fig. 6. This gives an explanation for the stationary distribution and the kinetics of evolution. We do not know, at this point in our investigation, if the two-dimensional network has the detailed dissipative structure of the simplified model, but we are able to produce evidence that the same argument employing the relative entropy does suggest the correct kinetics and stationary distribution.

\section{A. Formulation}

Let $I \subset \mathbf{R}$ be an interval of length $L$ partitioned by points $x_{i}, i=1, \ldots, n$, where $x_{i}<x_{i+1}, i=1, \ldots, n-1$ and $x_{n+1}$ is identified with $x_{1}$. For each interval $\left[x_{i}, x_{i+1}\right], i=1, \ldots, n$, select a random misorientation number $\alpha_{i} \in(-\pi / 4, \pi / 4]$. The intervals $\left[x_{i}, x_{i+1}\right]$ correspond to grain boundaries with misorientations $\alpha_{i}$ and the points $x_{i}$ represent the triple junctions. Choose an energy density $\psi(\alpha) \geqslant 0$ and introduce the energy

$$
E=\sum_{i=1, \ldots, n} \psi\left(\alpha_{i}\right)\left(x_{i+1}-x_{i}\right)
$$

We impose gradient flow kinetics with respect to (13), which is the system of ordinary differential equations

$$
\begin{gathered}
\frac{d x_{i}}{d t}=-\frac{\partial E}{\partial x_{i}}, i=1, \ldots, n, \text { that is } \\
\frac{d x_{i}}{d t}=\psi\left(\alpha_{i}\right)-\psi\left(\alpha_{i-1}\right), i=2, \ldots, n, \text { and } \\
\frac{d x_{1}}{d t}=\psi\left(\alpha_{1}\right)-\psi\left(\alpha_{n}\right) .
\end{gathered}
$$

The velocity $v_{i}$ of the $i$ th boundary is

$$
v_{i}=\frac{d x_{i+1}}{d t}-\frac{d x_{i}}{d t}=\psi\left(\alpha_{i-1}\right)-2 \psi\left(\alpha_{i}\right)+\psi\left(\alpha_{i+1}\right) .
$$

The grain boundary velocities are constant until one of the boundaries collapses. That segment is removed from the inventory of active cells, and the velocities of its two neighbors are changed due to the emergence of a new junction. Each such deletion event rearranges the network and, therefore, affects its subsequent evolution just as in the two-dimensional cellular network. Actually, since the interval velocities are constant, this gradient flow is just a sorting problem. At any time, the next deletion event occurs at the smallest of

$$
\frac{x_{i}-x_{i+1}}{v_{i}} \text { with } \quad v_{i}<0 .
$$

We turn to the dissipation inequality for the gradient flow. At any time $t$ between deletion events,

$$
\begin{aligned}
\frac{d E}{d t} & =\sum \psi\left(\alpha_{i}\right) v_{i} \\
& =-\sum\left[\psi\left(\alpha_{i}\right)-\psi\left(\alpha_{i-1}\right)\right]^{2} \\
& =-\sum \frac{d x_{i}{ }^{2}}{d t} \leqslant 0 .
\end{aligned}
$$

We may write a mass-spring-dashpot-like local dissipation inequality analogous to the grain growth one. In an interval $\left(t_{0}, t_{0}+\tau\right)$ where there are no critical events, $d E / d t$ may be 
integrated to give

$$
\tau \sum_{i=1, \ldots, n} \frac{d x_{i}^{2}}{d t}+E\left(t_{0}+\tau\right)=E\left(t_{0}\right)
$$

or

$$
\sum_{i=1, \ldots, n} \int_{0}^{\tau} \frac{d x_{i}^{2}}{d t} d t+E\left(t_{0}+\tau\right)=E\left(t_{0}\right) .
$$

With appropriate interpretation of the sum, (17) holds for all $t_{0}$ and almost every $\tau$ sufficiently small. With the obvious use of Young's inequality, ${ }^{37}$ we have that

$$
\frac{1}{4} \sum_{i=1, \ldots, n} \int_{0}^{\tau} v_{i}^{2} d t+E\left(t_{0}+\tau\right) \leqslant E\left(t_{0}\right) .
$$

The energy of the system at time $t_{0}+\tau$ is determined by its state at time $t_{0}$. Vice versa, changing the sign on the right-hand side of (14) allows us to begin with the state at time $t_{0}+\tau$ and return to the state of time $t_{0}$ : the system is reversible in an interval of time absent of rearrangement events. This is no longer the situation after such an event. At the later time, we have no knowledge about which interval, now no longer in the inventory, was deleted.

We introduce a new ensemble based on the misorientation parameter $\alpha$ where we take $\Omega:-\frac{\pi}{4}<\alpha<\frac{\pi}{4}$ for later ease of comparison with the two-dimensional network. The GBCD or character distribution in this context is, as expected, the histogram of lengths of intervals sorted by misorientation $\alpha$ scaled to be a probability distribution on $\Omega$. To be precise, let

$$
\begin{aligned}
l_{i}(\alpha, t)= & x_{i+1}(t)-x_{i}(t) \\
= & \text { length of the } i \text { th interval, } \\
& \text { where explicit note has been taken of } \\
& \text { its misorientation parameter } \alpha .
\end{aligned}
$$

Now partition $\Omega$ into $m$ subintervals of length $h=\frac{\pi}{2} \frac{1}{m}$, and let

$$
\begin{aligned}
& \rho(\alpha, t)=\sum_{\alpha^{\prime} \in((k-1) h, k h]} l_{i}\left(\alpha^{\prime}, t\right) \frac{1}{L h} \\
& \text { for }(k-1) h<\alpha \leqslant k h, t>0 .
\end{aligned}
$$

For this definition of the statistic,

$$
\int_{\Omega} \rho(\alpha, t) d \alpha=1 .
$$

Note that

$$
\begin{gathered}
\frac{\partial \rho}{\partial t}(\alpha, t)=\sum_{\alpha^{\prime} \in((k-1) h, k h]} v_{i}\left(\alpha^{\prime}\right) \frac{1}{L h} \\
\text { for }(k-1) h<\alpha \leqslant k h .
\end{gathered}
$$

We may express (18) in terms of the character distribution (19), which amounts to

$$
\begin{aligned}
& \mu_{0} \int_{t_{0}}^{t_{0}+\tau} \int_{\Omega}\left|\frac{\partial \rho}{\partial t}\right|^{2} d \alpha d t+\int_{\Omega} \psi(\alpha) \rho\left(\alpha, t_{0}+\tau\right) d \alpha \\
& \quad \leqslant \int_{\Omega} \psi(\alpha) \rho\left(\alpha, t_{0}\right) d \alpha
\end{aligned}
$$

where $\mu_{0}>0$ is some constant.
We now impose a modeling assumption. The expression (21) is in terms of the new misorientation level ensemble, upscaled from the local level of the original system. Consistent with the lack of reversibility when rearrangement events occur, an entropic term will be added. We use standard configurational entropy,

$$
+\int_{\Omega} \rho \log \rho d \alpha,
$$

although this is not the only choice. Minimizing (22) favors the uniform state, which would be the situation were $\psi(\alpha)=$ const.

Given that (21) holds, we assume for any $t_{0}$ and $\tau$ sufficiently small that

$$
\begin{aligned}
& \mu_{0} \int_{t_{0}}^{t_{0}+\tau} \int_{\Omega}\left(\frac{\partial \rho}{\partial t}\right)^{2} d \alpha d t+\left.\int_{\Omega}(\psi \rho+\lambda \rho \log \rho) d \alpha\right|_{t_{0}+\tau} \\
& \quad \leqslant\left.\int_{\Omega}(\psi \rho+\lambda \rho \log \rho) d \alpha\right|_{t_{0}} .
\end{aligned}
$$

$E(t)$ was analogous to an internal energy or the energy of a microcanonical ensemble, and now

$$
F(\rho)=F_{\lambda}(\rho)=E(t)+\lambda \int_{\Omega} \rho \log \rho d \alpha
$$

is a free energy.

\section{B. Fokker-Planck paradigm}

Equation (23) above fails as a proper dissipation principle because the first term does not represent lost energy due to frictional or viscous forces. For a deformation path $f(\alpha, t), t_{0} \leqslant$ $t \leqslant t_{0}+\tau$, of probability densities, this quantity is

$$
D=D(f)=\int_{0}^{\tau} \int_{\Omega} v^{2} f d \alpha d t,
$$

where $f, v$ are related by the continuity equation and initial and terminal conditions

$$
\begin{gathered}
f_{t}+(v f)_{\alpha}=0 \quad \text { in } \Omega \times\left(t_{0}, t_{0}+\tau\right) \text { and } \\
f\left(\alpha, t_{0}\right)=\rho\left(\alpha, t_{0}\right), \quad f\left(\alpha, t_{0}+\tau\right)=\rho\left(\alpha, t_{0}+\tau\right) \text { in } \Omega,
\end{gathered}
$$

by analogy with fluids, ${ }^{38}$ p. 53 et seq. and elementary mechanics.

For brevity, set $\rho^{*}(\cdot)=\rho\left(\cdot, t_{0}\right)$. Our question now is whether the first term of (23) can dominate the term $D(f)$ for some $f$ so that $D(f)$ may be substituted while maintaining the inequality. Using the deformation path given by $\rho$ itself, we may calculate that indeed

$$
\begin{aligned}
D(\rho) & =\int_{0}^{\tau} \int_{\Omega} v^{2} \rho d \alpha d t \\
& \leqslant \frac{c_{\Omega}}{\min _{\Omega} \rho} \cdot \int_{0}^{\tau} \int_{\Omega}\left(\frac{\partial \rho}{\partial t}\right)^{2} d \alpha d t,
\end{aligned}
$$

where the $v$ is chosen by solving explicitly the continuity equation (26). We now have that for any relaxation time $\tau>0$,

$$
\frac{\mu}{2} \int_{0}^{\tau} \int_{\Omega} v^{2} \rho d \alpha d t+F_{\lambda}(\rho) \leqslant F_{\lambda}\left(\rho^{*}\right)
$$

for some constant $\mu$. 
The infimum of $D(f)$ over all admissible $(v, f)$ is a known statistical measure of closeness of probability densities, the square of the Kantorovich-Rubinstein-Wasserstein or Wasserstein metric. ${ }^{39,40}$ For densities $\rho, \rho^{*}$ it is defined to be

$$
\begin{gathered}
d\left(\rho, \rho^{*}\right)^{2}=\inf _{P} \int_{\Omega} \int_{\Omega}|x-y|^{2} d p(x, y), \\
P=\text { joint distributions for } \rho, \rho^{*} \text { on } \bar{\Omega} \times \bar{\Omega} .
\end{gathered}
$$

Recall here that the probability density $p(x, y)$ is a joint distribution for the probability distributions $P$ and $P^{*}$ with densities $\rho$ and $\rho^{*}$ provided that

$$
\begin{gathered}
P(E)=\int_{E} \rho(x) d x=\int_{E} \int_{\Omega} d p(x, y) \text { and } \\
P^{*}(F)=\int_{F} \rho^{*}(y) d y=\int_{\Omega} \int_{F} d p(x, y) \\
\text { for all } E, F \subset \Omega .
\end{gathered}
$$

The metric $d$ has the property that

$$
\frac{1}{\tau} d\left(\rho, \rho^{*}\right)^{2}=\inf D(f),
$$

where the infimum is taken over all deformation paths $(f, v)$ satisfying (26). ${ }^{41}$ We next replace $(28)$ by a minimum principle, arguing that the path given by $\rho(\alpha, t)$ is the one most likely to occur and that the minimizing path has the highest probability. We are led to the variational principle for the unknown $\rho$ given $\rho^{*}$,

$$
\frac{\mu}{2 \tau} d\left(\rho, \rho^{*}\right)^{2}+F_{\lambda}(\rho)=\inf _{\{\eta\}}\left\{\frac{\mu}{2 \tau} d\left(\eta, \rho^{*}\right)^{2}+F_{\lambda}(\eta)\right\} .
$$

For each relaxation time $\tau>0$ we determine iteratively the sequence $\left\{\rho^{(k)}\right\}$ by choosing $\rho^{*}=\rho^{(k-1)}$ and $\rho^{(k)}=\rho$ in (31), and we set

$$
\rho^{(\tau)}(\alpha, t)=\rho^{(k)}(\alpha) \text { in } \Omega \text { for } \quad k \tau \leqslant t<(k+1) \tau .
$$



We then anticipate recovering the $\operatorname{GBCD} \rho$ as

$$
\rho(\alpha, t)=\lim _{\tau \rightarrow 0} \rho^{(\tau)}(\alpha, t),
$$

with the limit taken in a suitable sense. ${ }^{42}$ It has been recently established that $\rho$ obtained from (33) is the solution of the Fokker-Planck equation, ${ }^{10}$

$$
\mu \frac{\partial \rho}{\partial t}=\frac{\partial}{\partial \alpha}\left(\lambda \frac{\partial \rho}{\partial \alpha}+\psi^{\prime} \rho\right) \quad \text { in } \quad \Omega, \quad 0<t<\infty .
$$

We might point out here, as well, that a solution of (34) with periodic boundary conditions and non-negative initial data is positive for $t>0$.

\section{VALIDATION OF THE SCHEME}

We now begin the validation step of our model. First we review a few facts about solutions of (34). Introduce the notation for the Boltzmann distribution with parameter $\lambda$,

$$
\begin{gathered}
\rho_{\lambda}(\alpha)=\frac{1}{Z_{\lambda}} e^{-\frac{1}{\lambda} \psi(\alpha)}, \alpha \in \Omega, \quad \text { where } \\
Z_{\lambda}=\int_{\Omega} e^{-\frac{1}{\lambda} \psi(\alpha)} d \alpha .
\end{gathered}
$$

The Kullback-Leibler relative entropy with parameter $\lambda$ for (34) is given by

$$
\begin{gathered}
\Phi_{\lambda}(\eta)=\lambda \int_{\Omega} \eta \log \frac{\eta}{\rho_{\lambda}} d \alpha, \quad \text { where } \\
\eta \geqslant 0 \text { in } \Omega, \int_{\Omega} \eta d \alpha=1,
\end{gathered}
$$

with $\rho_{\lambda}$ from (35). It is a convex function of $\eta$, and by Jensen's inequality it is always non-negative. ${ }^{43}$ In terms of the free energy (24) and (35), (36) is given by

$$
\Phi_{\lambda}(\eta)=F_{\lambda}(\eta)+\lambda \log Z_{\lambda}
$$

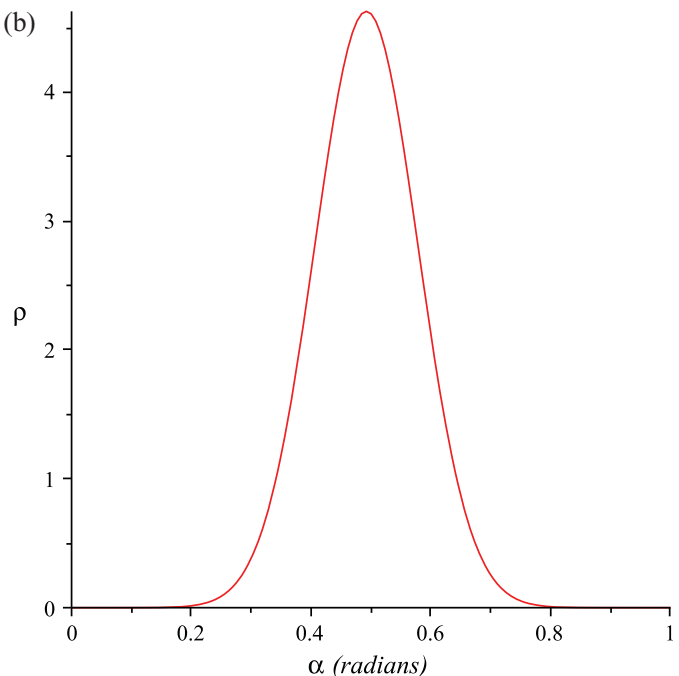

FIG. 4. (Color online) (a) The relative entropy $\Phi_{\sigma}$ of the solution $u(x, t)$ of the Fokker-Planck equation (34) for the potential $\psi(x)=$ $1+r\left(x-\frac{1}{2}\right)^{2}, r=2$, with the choice $\lambda=\sigma=0.0296915$, computed by a routine numerical method, compared with a sequence of $\Phi_{\lambda}$ with the curve for $\sigma=0.0296915$ noted in red. The values of $\lambda$ correspond to $\rho_{\lambda}$ with $\max \rho_{\sigma} / 2 \leqslant \max \rho_{\lambda} \leqslant(3 / 2)$ max $\rho_{\sigma}$. (b) The computed equilibrium solution, which is indistinguishable from $\rho_{\sigma}$, the Boltzmann distribution of (36). 


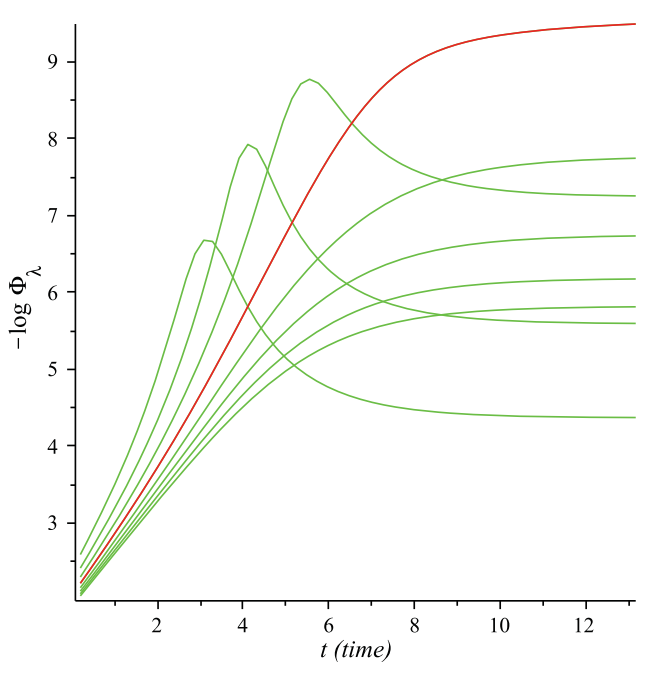

FIG. 5. (Color online) Plots of $-\log \Phi_{\lambda}$ vs $t$ with $-\log \Phi_{\sigma}$ in red for the solution of (34), cf. Fig. 4. The plot illustrates that $\Phi_{\sigma}$ decreases exponentially to 0 but that $\Phi_{\lambda}$ for choices of $\lambda \neq \sigma$ do not have this property.

that is, it differs from the free energy by a known function of $\lambda$. A solution $\rho$ of (34) with $\lambda=\sigma$ satisfies, ${ }^{40}$ or $^{13}$ for an elementary demonstration,

$$
\begin{gathered}
\lim _{t \rightarrow \infty} \Phi_{\sigma}(\rho)=0 \text { exponentially fast, whereas } \\
\lim _{t \rightarrow \infty} \Phi_{\lambda}(\rho)>0 \text { for } \lambda \neq \sigma .
\end{gathered}
$$

From (38) and the classical Csiszar-Kullback inequality, ${ }^{40,44}$

$$
\rho(\alpha, t) \rightarrow \rho_{\sigma}(\alpha) \text { as } t \rightarrow \infty \text { exponentially fast. }
$$

We point out here that (39) follows whenever a function satisfies (38).

We now turn to the validation of our method. The procedure that leads to the implicit scheme is based on a dissipation inequality, (18), that holds for the entire system but does not

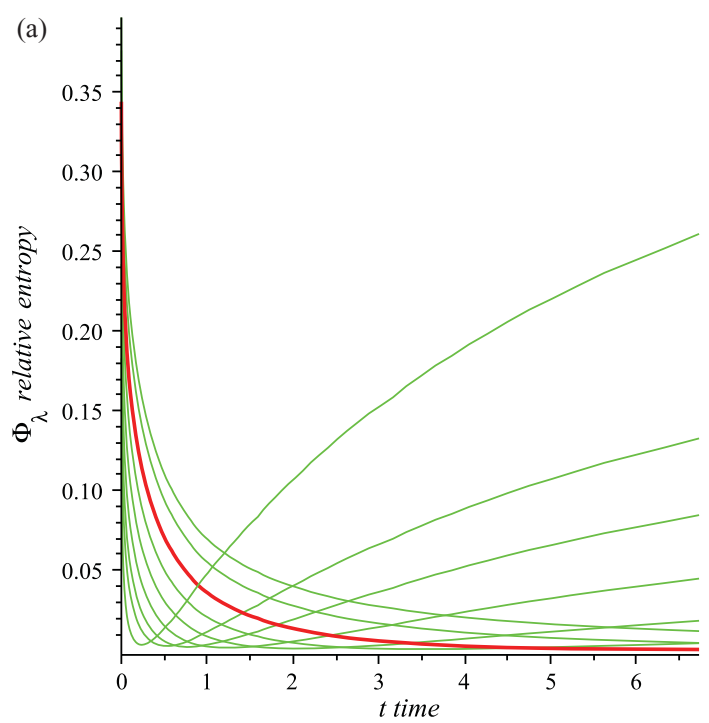

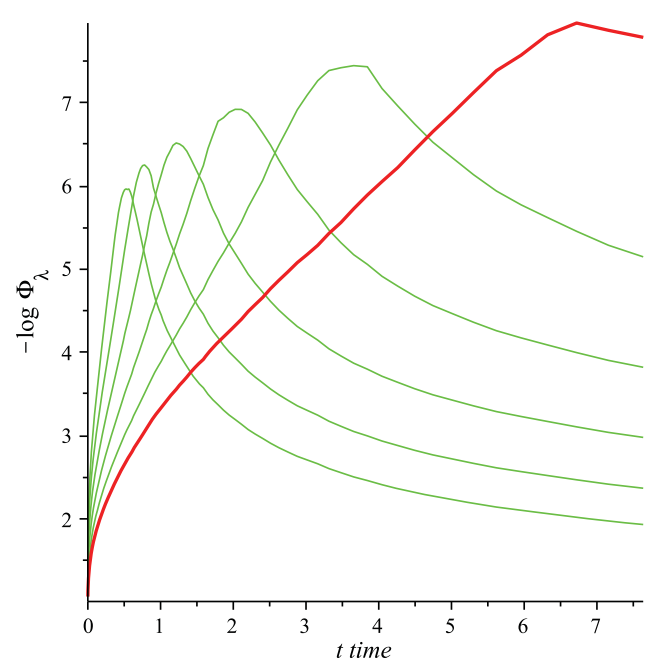

FIG. 7. (Color online) Plots of $-\log \Phi_{\lambda}$ vs $t$ with $-\log \Phi_{\sigma}, \sigma=$ 0.0296915 , in red for the simplified coarsening model with potential (40). It shows that $\Phi_{\sigma}$ decays exponentially to its minimum at simulation time $t=T_{\infty}$.

identify individual intermediate "spring-mass dashpots." The consequence is that we cannot set the parameter $\sigma$, but in some way must decide if one exists, as we have been suggesting.

Therefore, we seek to identify the particular $\lambda=\sigma$ for which $\Phi_{\sigma}$ defined by the GBCD statistic $\rho$ tends monotonically to the minimum of all the $\left\{\Phi_{\lambda}\right\}$ as $t$ becomes large. The empirical GBCD $\rho$ is a statistic, and so the minimum of all the $\left\{\Phi_{\lambda}\right\}$ may not be zero. So we must proceed to ask if the terminal, or equilibrium, empirical distribution $\rho$ is equal, that is, reasonably close, to $\rho_{\sigma}$ given by the formula (35). This is the essence of our validation procedure. For our purposes, we simply decide the question of equality by inspection.

With validation, we would gain qualitative properties of solutions of (34): If we find the correct choice for $\lambda=\sigma$, then from our discussion above,

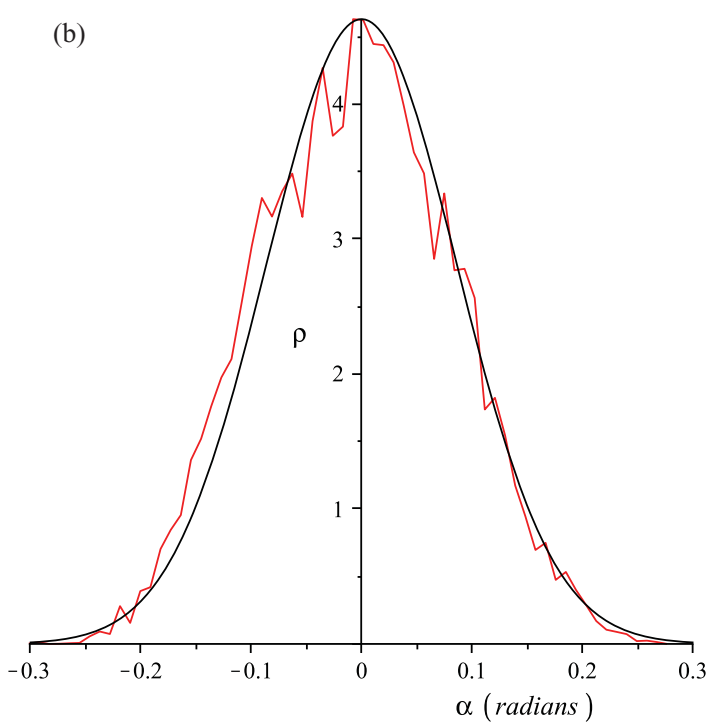

FIG. 6. (Color online) Graphical results for the simplified coarsening model with potential (40). (a) Relative entropy plots for values of $\lambda$ chosen according to (41) with $\Phi_{\sigma}$ noted in red. The value of $\sigma=0.029691$ 5. (b) Empirical GBCD at simulation time $t=T_{\infty}$ in red compared with $\rho_{\sigma}$ in black. 
(i) $\rho(\alpha, t) \rightarrow \rho_{\sigma}(\alpha)$ as $t \rightarrow \infty$, and

(ii) this convergence is exponentially fast, and otherwise these properties fail.

In this context, determining a parameter on the basis of its thermodynamic restrictions is well known. A noteworthy feature here is its use in a nonequilibrium setting; cf. also Refs. 45-47.

To understand our implementation, we offer an illustration using the solution of (34) itself, $u$ computed on $\Omega=(0,1)$ with the choices $\psi(x)=1+r(x-1 / 2)^{2}, r=2$, and $\lambda=$ $\sigma=0.0296915$, and a collection of relative entropy plots $\left\{\Phi_{\lambda}\right\}$ where values of $\lambda$ are close to $\sigma$, cf. Fig. 4(a). The plot of $\Phi_{\sigma}$ vs time $t$ is noted in red and it is decreasing and tends to 0 . A glance at the resulting equilibrium $u$, Fig. 4(b), identifies it as the Boltzmann distribution $\rho_{\sigma}$, as constructed. In Fig. 5, plots of $-\log \Phi_{\lambda}$ are shown, illustrating that $-\log \Phi_{\sigma}$ increases linearly, or $\Phi_{\sigma}$ decreases exponentially while $\Phi_{\lambda}$ for $\lambda \neq \sigma$ does not have this property.

For the simplified coarsening model, we consider

$$
\psi(\alpha)=1+2 \alpha^{2}, \quad \alpha \in \Omega=\left(-\frac{\pi}{4}, \frac{\pi}{4}\right),
$$

and we shall identify one such unique parameter, which we label $\sigma$, by seeking the minimum of the relative entropy (36) and then comparing it with $\rho_{\sigma}$. This $\psi$ is the development to second order of $\psi(\alpha)=1+0.5 \sin ^{2} 2 \alpha$ used in the two-dimensional simulation. Moreover, since the potential is quadratic, it represents a version of the Ornstein-Uhlenbeck process ${ }^{48}$ that we computed above directly. To proceed, we must agree upon which simulation time $t=T_{\infty}$ represents time equal to infinity. For the simplified critical event model we are considering, it is clear that by computing for a sufficiently long time, all cells will be gone. This time may be quite long. We choose the time parameter so that $80 \%$ of segments have been deleted, which corresponds to the stationary configuration in the twodimensional simulation. For the simplified model simulation, this time is $T_{\infty}=T(80 \%)=6.73$. Here, $T(\Xi)$ denotes the time at which $\Xi \%$ of the cells have been deleted. For comparison,
$T(90 \%)=30$ and $T(95 \%)=103$. There may be additional criteria for choosing a terminal time $T_{\infty}$ in the neighborhood of $T(80 \%)$, and we may wish to discuss this later.

This simulation is initialized with $2^{15}+1$ cells, and approximately 155 trial distributions $\rho_{j}$ are collected at 200 rearrangement event intervals. A total of 155 trial relative entropies are constructed from Gaussians $\rho_{\lambda_{j}}$ satisfying

$$
\rho_{\lambda_{j}}(0)=\max \rho_{\lambda_{j}}=\max \rho_{j} .
$$

Some of these are shown in Fig. 6(a). The empirical GBCD is compared with the appropriate Boltzmann distribution in Fig. 6(b).

We include the plots of $-\log \Phi_{\lambda}$, Fig. 7, which suggests that $\Phi_{\sigma}$ decays exponentially to its minimum whereas $\Phi_{\lambda}$ corresponding to other values of $\lambda$ do not.

For a second example to illustrate the method, we consider the potential

$$
\psi(\alpha)=1+\epsilon \alpha^{4}, \alpha \in \Omega, \epsilon=8 .
$$

This choice, $\epsilon=8$, corresponds to the first-order terms in the two-dimensional quartic energy density we discuss in the next section. Also here in Fig. 8 one sees very good agreement between the empirical GBCD and the appropriate Boltzmann distribution.

\section{THE ENTROPY METHOD FOR THE GBCD}

We shall apply the method of Sec. IV to the GBCD harvested from the 2D simulation. We consider first a typical simulation with the energy density

$$
\psi(\alpha)=1+\epsilon(\sin 2 \alpha)^{2}, \quad-\frac{\pi}{4} \leqslant \alpha \leqslant \frac{\pi}{4}, \quad \epsilon=1 / 2,
$$

Fig. 9, initialized with $10^{4}$ cells and normally distributed misorientation angles and terminated when 2000 cells remain. At this stage, the simulation is essentially stagnant. Possible
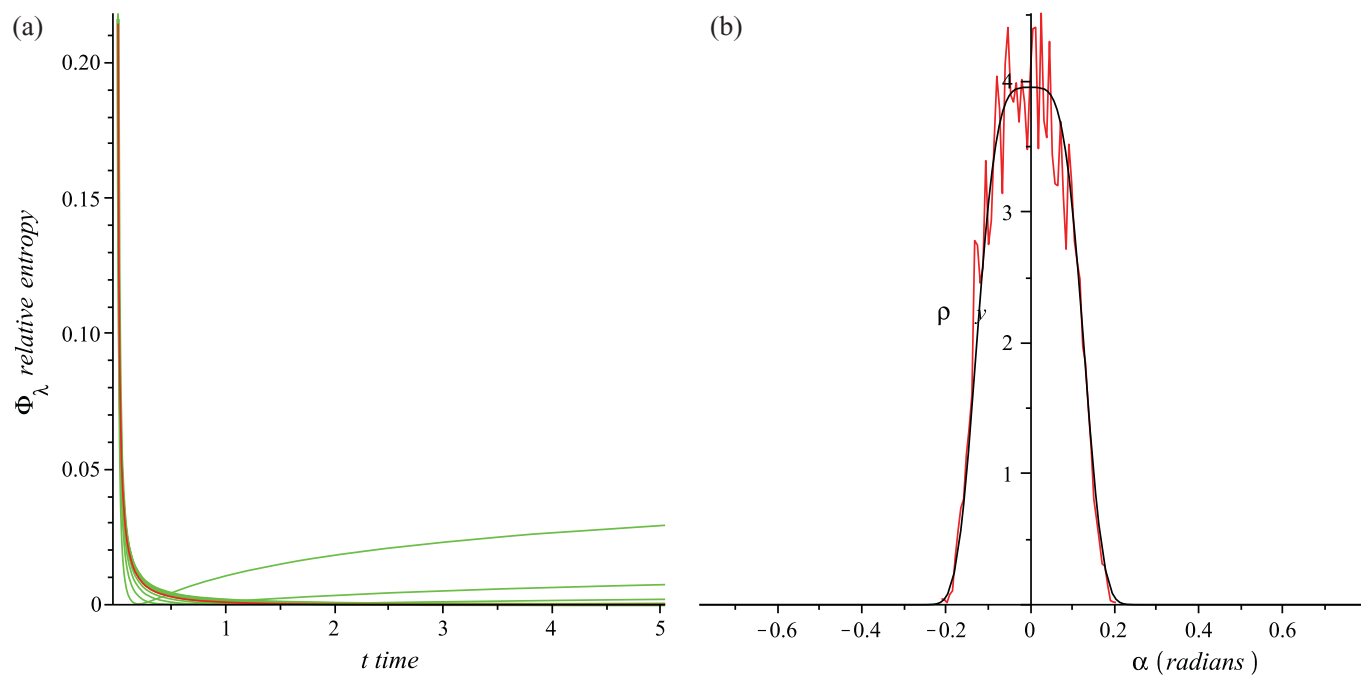

FIG. 8. (Color online) Graphical results for the simplified coarsening model with potential (42). (a) Relative entropy plot for selected values of $\lambda$ with $\Phi_{\sigma}$ noted in red. The value of $\sigma=0.003033356683$ and is ascertained at the simulation time $t=T_{\infty}$ corresponding to $80 \%$ of cells deleted. (b) Empirical GBCD at time $t=T_{\infty}$ in red compared with $\rho_{\sigma}$ in black. 




FIG. 9. (Color online) The energy density $\psi(\alpha)=1+$ $\epsilon \sin ^{2} 2 \alpha,|\alpha|<\pi / 4, \epsilon=\frac{1}{2}$.

parameters $\lambda$ are constructed similarly to those of the simplified coarsening model: From the maximum of a harvested GBCD, we construct the Gaussian with the same maximum. This determines a value of $\lambda$ that is used to define $\rho_{\lambda}$ in (35) for the density (43). This $\rho_{\lambda}$ then defines a trial relative entropy via (36).

We now identify the parameter $\sigma$, which turns out to be $\sigma \approx 0.1$, Fig. 10(a). In Fig. 10(b) the empirical GBCD is compared with the Boltzmann distribution with the parameter determined by Fig. 10(a), showing excellent agreement. From Fig. 11, we see that this relative entropy $\Phi_{\sigma}$ has exponential decay until it reaches a value of about 1.5, when it remains constant. The solution itself thus tends exponentially (in $L^{1}$ ) to its limit $\rho_{\sigma}$ by the Csiszar-Kullback inequality.

Figure 12 shows that averaging over a few trials, five in this case, the empirical GBCD's approach the Boltzmann distribution $\rho_{\sigma}$ of (35) quite closely.

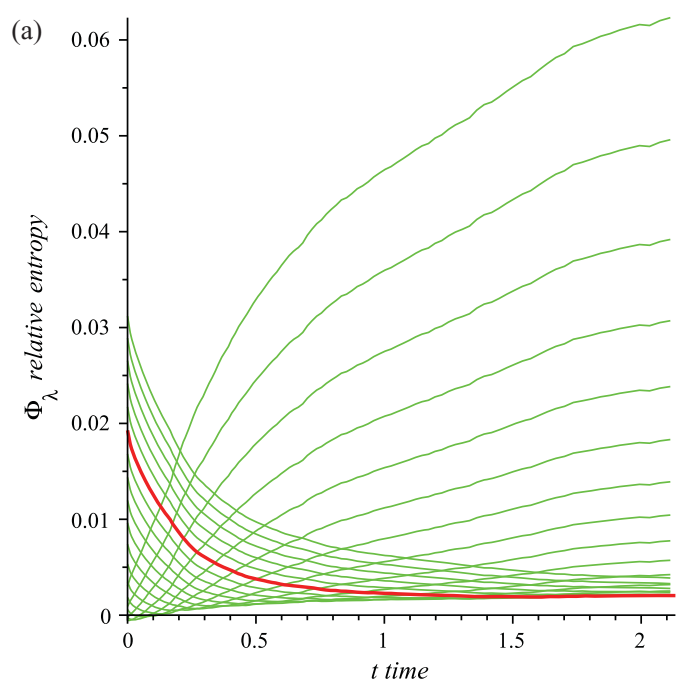

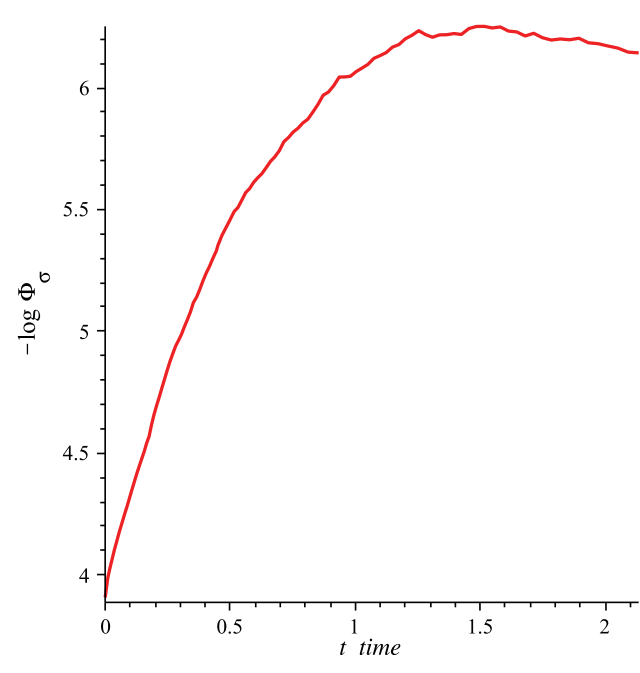

FIG. 11. (Color online) Plot of $-\log \Phi_{\sigma}$ vs $t$ with energy density (43). It is approximately linear until it becomes constant showing that $\Phi_{\sigma}$ decays exponentially.

A second example presented here is a quartic energy,

$$
\psi(\alpha)=1+\epsilon(\sin 2 \alpha)^{4}, \quad-\frac{\pi}{4} \leqslant \alpha \leqslant \frac{\pi}{4}, \quad \epsilon=1 / 2 .
$$

Again, a configuration of $10^{4}$ cells is initialized with normally distributed misorientations, and, this time, the computation proceeds until about 1000 cells remain. The relative entropy and the equilibrium Boltzmann statistic stabilize when 2000 cells remain.

With the equilibrium solution in hand, as depicted in Fig. 13, we again initialized a configuration of $10^{4}$ cells with, on this occasion, misorientations normally distributed in the much narrower range defined by the sides of the solution GBCD. Since these misorientations see, essentially, only the near minimum of the potential, we would expect the new stationary distribution to be Gaussian or random. However, we obtain

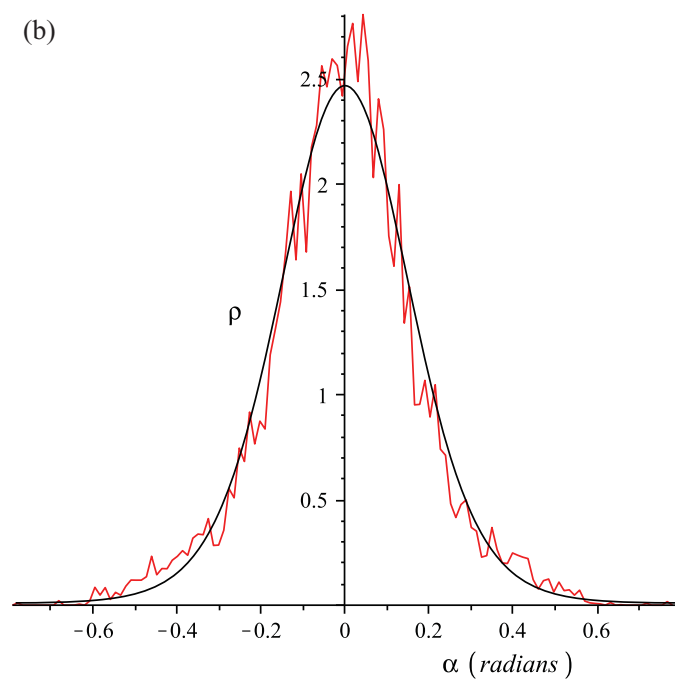

FIG. 10. (Color online) (a) The relative entropy of the grain growth simulation with energy density (43) for a sequence of $\Phi_{\lambda}$ vs $t$ with the optimal choice $\sigma \approx 0.1$ noted in red. (b) Comparison of the empirical GBCD distribution at time $t=T_{\infty}=2$, when $80 \%$ of the cells have been deleted, with $\rho_{\sigma}$ the Boltzmann distribution of (35). 


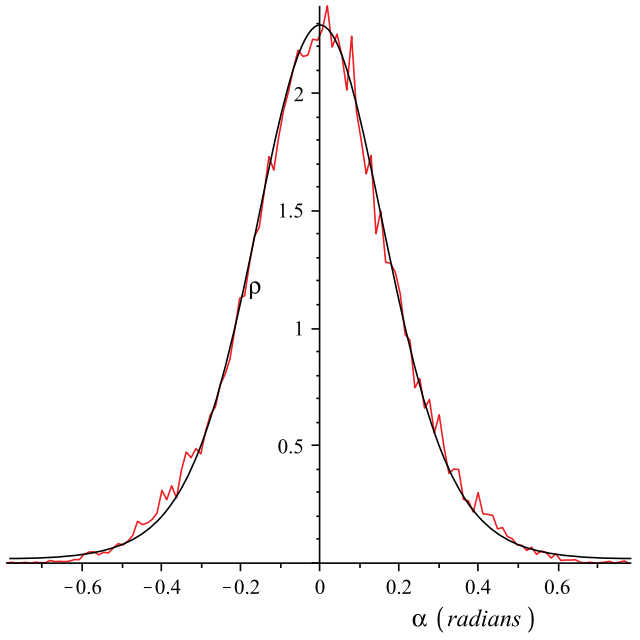

FIG. 12. (Color online) GBCD (red) and Boltzmann distribution (black) for the potential $\psi$ of (43) with parameter $\sigma \approx 0.1$ as predicted by our theory. This GBCD is averaged over five trials.

the same relative entropy curve and equilibrium depicted in Fig. 13. Although coarsening is not like a molecular system with eternal collisions causing the entire system to equilibrate, the fluctuations of misorientations caused by the "perpetual" critical events provide the system with a sufficiently ample library to be driven by the given grain boundary energy density. On the other hand, we may defeat this attribute, for example, with a Read-Shockley type of energy, which is cusplike near the origin and rises sharply to a maximum. Near the origin, we obtain a reasonable distribution, however there are otherwise insufficient orientations to populate a Boltzmann distribution. ${ }^{16}$

Future work will address the theory when the interfacial energy density $\psi=\psi(\theta, \alpha)$ depends on both normal angle and misorientation of the interface. In this context, we have ob- served that simply resolving the solution of the Fokker-Planck equation with quartic potential leads to bimodal intermediate distributions, which are the stationary distributions for quartic interfacial energy distributions. ${ }^{3,49}$ This suggests that this situation represents the quenched solution of a Fokker-Planck equation and a role for the second eigenfunction of the equation. Other effects will also be studied. These can be added to the local evolution law, most simply varying mobility, and other retarding forces such as triple junction drag.

\section{DISCUSSION AND CONCLUSIONS}

We have outlined an entropy-based theory of the GBCD that is an upscaling of cell growth according to the two most basic properties of a coarsening network: a local evolution law and space-filling constraints. The theory accommodates the irreversibility conferred by the critical events or topological rearrangements that arise during coarsening. Details are given for a model system in which the analytical tools are easily exploited, and they are seen to describe well the results of two-dimensional simulations. Our principal conclusion is that these events occur preferentially in a manner that renders the GBCD closely related to the solution of a Fokker-Planck equation whose potential is the given interfacial energy density. This reasoning exploits the recent characterization of FokkerPlanck kinetics as a gradient flow for the free energy.

We note that the theory states in particular that it is the GBCD that is a consequence of the coarsening process. The traditional texture distribution is the orientation distribution (OD), the distribution of grain orientations. The GBCD is the distribution of differences of the OD, basically the convolution of the OD with itself. This relationship may be inverted by elementary Fourier analysis, so, in this simple case, the GBCD determines the OD and not the other way around. Therefore, we may expect, in nature, that it is among the processes that determine the OD.
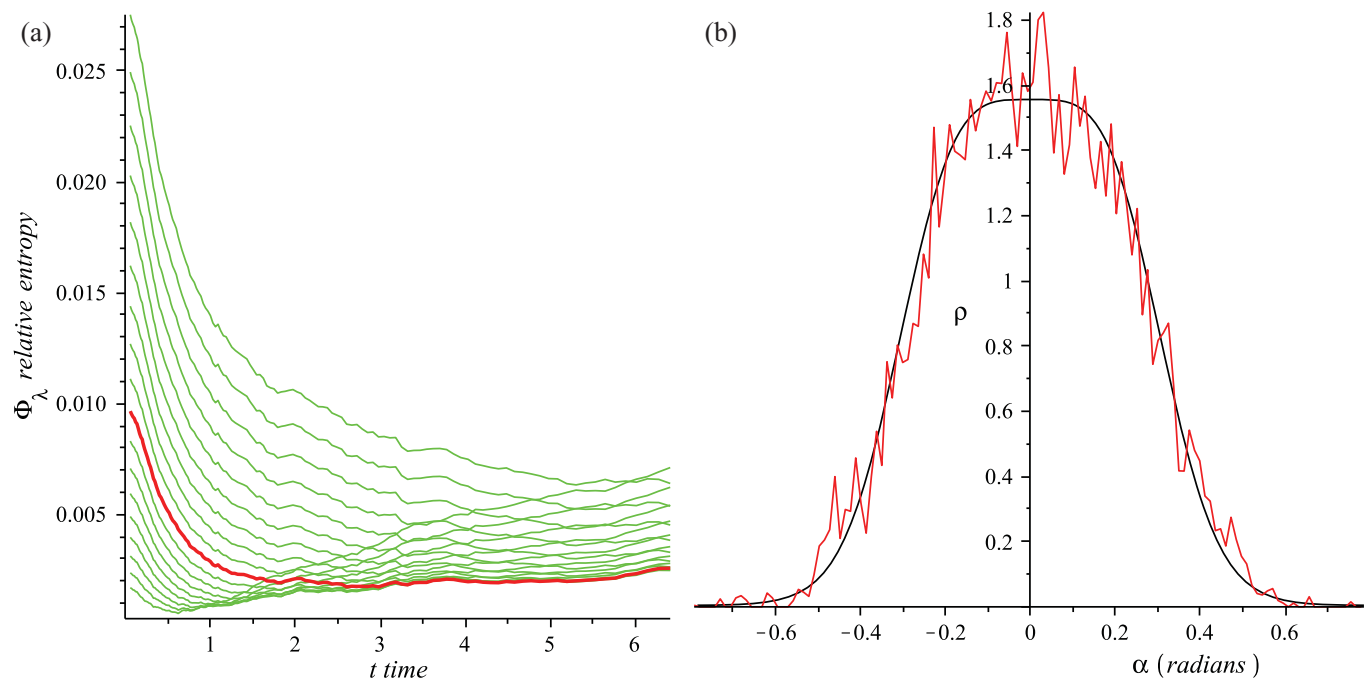

FIG. 13. (Color online) (a) The relative entropy of the grain growth simulation with density (44) for a sequence of $\Phi_{\lambda}$ vs $t$ with the optimal choice $\sigma \approx 0.08$ noted in red. (b) Comparison of the empirical GBCD at time $t=T_{\infty}=3$, when $80 \%$ of the cells have been deleted, with $\rho_{\sigma}$ the Boltzmann distribution of (35). 


\section{ACKNOWLEDGMENTS}

This research was done while Y.E. and R.S. were postdoctoral associates at the Center for Nonlinear Analysis. We are grateful to our colleagues G. Rohrer, A. D. Rol- lett, R. Schwab, and R. Suter, for their collaboration. Research was supported by NSF DMR0520425, DMS 0405343, DMS 0305794, DMS 0806703, DMS 0635983, and DMS 0915013. *katayun@andrew.cmu.edu

$\dagger$ eva.eggeling@fraunhofer.at

${ }_{\ddagger}^{\ddagger}$ memelian@gmu.edu

§epshteyn@math.utah.edu

"davidk@andrew.cmu.edu

Trsharp@gmail.com

***hlomo@andrew.cmu.edu

${ }^{1}$ B. L. Adams, D. Kinderlehrer, W. W. Mullins, A. D. Rollett, and S. Ta'asan, Scr. Mater. 38, 531 (1998).

${ }^{2}$ B. L. Adams, D. Kinderlehrer, I. Livshits, D. Mason, W. W. Mullins, G. S. Rohrer, A. D. Rollett, D. Saylor, S Ta'asan, and C. Wu, Interface Sci. 7, 321 (1999).

${ }^{3}$ D. Kinderlehrer, I. Livshits, G. S. Rohrer, S. Ta'asan, and P. Yu, Mater. Sci. Forum 467-470, 1063 (2004).

${ }^{4}$ G. S. Rohrer, Annu. Rev. Mater. Res. 35, 99 (2005).

${ }^{5}$ A. D. Rollett, S.-B. Lee, R. Campman, and G. S. Rohrer, Annu. Rev. Mater. Res. 37, 627 (2007).

${ }^{6} \mathrm{C}$. S. Smith, Grain Shapes and Other Metallurgical Applications of Topology, in Ref. 55, pp. 65-108.

${ }^{7}$ W. W. Mullins, Solid Surface Morphologies Governed by Capillarity, in Metal Surfaces: Structure, Energetics, and Kinetics (American Society for Metals, Metals Park, OH, 1963), pp. 17-66.

${ }^{8} \mathrm{C}$. Herring, Surface Tension as a Motivation for Sintering, in The Physics of Powder Metallurgy, edited by W. E. Kingston (McGrawHill, New York, 1951), pp. 143-179.

${ }^{9}$ See Ref. 50, p. 6.

${ }^{10}$ R. Jordan, D. Kinderlehrer, and F. Otto, SIAM J. Math. Anal. 29, 1 (1998).

${ }^{11}$ R. Jordan, D. Kinderlehrer, and F. Otto, Physica D 107, 265 (1997).

${ }^{12}$ K. Barmak, E. Eggeling, M. Emelianenko, Y. Epshteyn, D. Kinderlehrer, and S. Ta' asan, Rend. Matematica, Ser. VII 29, 65 (2009).

${ }^{13}$ K. Barmak, E. Eggeling, M. Emelianenko, Y. Epshteyn, D. Kinderlehrer, R. Sharp, and S. Ta'asan, Discrete Cont. Dyn. Sys. A 30(2), 427 (2011).

${ }^{14}$ K. Barmak, D. Kinderlehrer, I. Livshits, and S. Ta'asan, Remarks on a Multiscale Approach to Grain Growth in Polycrystals, in Variational Problems in Materials Science, Progress in Nonlinear Differential Equations Applications Vol. 68, edited by Gianni dal Maso, Antonio DeSimone, and Franco Tomarelli (Birkhäuser, Basel, 2006), pp. 1-11.

${ }^{15}$ A. Cohen, Multiscale Model. Simul. 8, 463 (2009/10).

${ }^{16}$ K. Barmak, E. Eggeling, M. Emelianenko, Y. Epshteyn, D. Kinderlehrer, R. Sharp, and S. Ta' asan, Predictive Theory for the Grain Boundary Character Distribution, in Proceedings of Recrystallization and Grain Growth IV (Materials Science Forum, Trans Tech Publications, Switzerland) (in press).

${ }^{17}$ J. Gruber, H. M. Miller, T. D. Hoffmann, G. S. Rohrer, and A. D. Rollett, Acta Mater. 57, 6102 (2009).

${ }^{18}$ J. Gruber, A. D. Rollett, and G. S. Rohrer, Acta Mater. 58, 14 (2010).

${ }^{19}$ E. A. Holm, G. N. Hassold, and M. A. Miodownik, Acta Mater. 49, 2981 (2001).
${ }^{20}$ M. A. Miodownik, P. Smereka, D. J. Srolovitz, and E. A. Holm, Proc. R. Soc. London, Ser. A 457, 1807 (2001).

${ }^{21} \mathrm{C}$. Herring, The Use of Classical Macroscopic Concepts in Surface Energy Problems, in Structure and Properties of Solid Surfaces, edited by R. Gomer and C. S. Smith (The University of Chicago Press, Chicago, 1952), pp. 5-81, proceedings of a conference arranged by the National Research Council and held in September, 1952, in Lake Geneva, WI, USA.

${ }^{22} \mathrm{M}$. Gurtin, Thermomechanics of Evolving Phase Boundaries in the Plane (Oxford University Press, Oxford, 1993).

${ }^{23}$ L. Bronsard and F. Reitich, Arch. Ration. Mech. Anal. 124, 355 (1993).

${ }^{24}$ D. Kinderlehrer and C. Liu, Math. Models Methods Appl. Sci. 11, 713 (2001)

${ }^{25}$ W. W. Mullins, Scr. Metall. 22, 1441 (1988).

${ }^{26}$ D. Kinderlehrer, I. Livshits, and S. Ta'asan, SIAM J. Sci. Comput. 28, 1694 (2006).

${ }^{27}$ D. Kinderlehrer, J. Lee, I. Livshits, A Rollett, and S. Ta' asan, Mater. Sci. Forum 467-470, 1057 (2004).

${ }^{28}$ J. von Neumann, discussion concerning the paper of C. S. Smith, Grain Shapes and Other Metallurgical Applications of Topology, in Ref. 55, pp. 108-110.

${ }^{29}$ W. W. Mullins, J. Appl. Phys. 27, 900 (1956).

${ }^{30}$ That is, the special case of $(3)^{8,21}$ when the interfacial energy density is independent of the normal direction; cf. Ref. 51.

${ }^{31}$ Perhaps it is also of value to point out, in this context, that the failure of all but one cell to evacuate the network under (10) cannot be attributed to an inability to form a triple junction, but is likely due to configurational hinderance or near-equilibrium behavior.

${ }^{32}$ The details of the preparation of the Al films (nominally $100 \mathrm{~nm}$ in thickness), their examination by transmission electron microscopy, and the methodology for obtaining the tracings of the grain boundary network are given in Ref. 52. From the grain boundary tracings, the number of sides and the size of each grain was determined. The grains were then sorted by their number of sides, and the average area of each side class was determined as a function of annealing time. Only the data for the six-sided grains are plotted in the figure here. All side classes between 3 and 9 showed the same trend.

${ }^{33}$ The simulation is based on the algorithms described in Refs. 26 and 27 mentioned in the preceding section.

${ }^{34}$ K. Barmak, M. Emelianenko, D. Golovaty, D. Kinderlehrer, and S. Ta'asan, SIAM J. Sci. Comput. 30, 3150 (2007).

${ }^{35}$ K. Barmak, M. Emelianenko, D. Golovaty, D. Kinderlehrer, and S. Ta'asan, Int. J. Numer. Anal. Modeling 5, 93 (2008).

${ }^{36} \mathrm{~K}$. Barmak, M. Emelianenko, D. Golovaty, D. Kinderlehrer, and S. Ta'asan, On a Statistical Theory of Critical Events in Microstructural Evolution, in Proceedings CMDS 11 (ENSMP Press, Paris, 2007), pp. 185-194.

${ }^{37} 2 a b \leqslant a^{2}+b^{2}$.

${ }^{38}$ L. D. Landau and E. M. Lifshitz, Fluid Mechanics, translated from the Russian by J. B. Sykes and W. H. Reid, Course of Theoretical Physics (Pergamon, London, 1959), Vol. 6, pp. xii+536. 
${ }^{39}$ L. Ambrosio, N. Gigli, and G. Savaré, Gradient Flows in Metric Spaces and in the Space of Probability Measures, 2nd ed., Lectures in Mathematics ETH Zürich (Birkhäuser Verlag, Basel, 2008).

${ }^{40}$ C. Villani, Topics in Optimal Transportation, Graduate Studies in Mathematics Vol. 58 (American Mathematical Society, Providence, RI, 2003).

${ }^{41}$ J.-D. Benamou and Y. Brenier, Numer. Math. 84, 375 (2000).

${ }^{42}$ Iterative schemes are among the most common ways of solving differential equations. Generally, they consist of metrics in competition with functionals. The best known method for solving the diffusion equation, the backward Euler or implicit finite-difference scheme,$^{53,54}$ is based on the variational principle for the unknown $u$ given $u^{*}$ :

$$
\frac{1}{2 \tau} \int_{\Omega}\left|u-u^{*}\right|^{2} d x+\frac{k}{2} \int_{\Omega}|\nabla u|^{2} d x=\min .
$$

The $L^{2}$ metric is in competition with the Dirichlet integral. For each relaxation time $\tau>0$, determine the sequence $\left\{u^{k}\right\}$ by choosing $u^{*}=u^{(k-1)}$ and $u=u^{(k)}$ in the variational principle above. Then define $u^{(\tau)}$ by

$$
u^{(\tau)}(x, t)=u^{(k)}(x) \text { in } \Omega, k \tau \leqslant t<(k+1) \tau,
$$

and let

$$
u=\lim _{\tau \rightarrow 0} u^{(\tau)} .
$$

Then $u$ is a solution of the diffusion equation

$$
\frac{\partial u}{\partial t}=k \Delta u \text { in } \Omega \text {. }
$$

${ }^{43}$ The function $\varphi(r)=r \log r$ is convex, hence

$$
0=\varphi(1)=\varphi\left(\int_{\Omega} \frac{\eta}{\rho_{\lambda}} \rho_{\lambda} d \alpha\right) \leqslant \int_{\Omega} \varphi\left(\frac{\eta}{\rho_{\lambda}}\right) \rho_{\lambda} d \alpha=\Phi_{\lambda}(\eta) .
$$

${ }^{44}$ S. Kullback, Information Theory and Statistics (Dover, Mineola, NY, 1997), pp. xvi+399, reprint of the second (1968) edition.

${ }^{45}$ E. Bouchbinder and J. S. Langer, Phys. Rev. E 80, 031131 (2009).

${ }^{46}$ E. Bouchbinder and J. S. Langer, Phys. Rev. E 80, 031132 (2009).

${ }^{47}$ E. Bouchbinder and J. S. Langer, Phys. Rev. E 80, 031133 (2009).

${ }^{48}$ N. G. van Kampen, Stochastic Processes in Physics and Chemistry (Elsevier, Amsterdam, 1992).

${ }^{49}$ D. M. Saylor, A. Morawiec, and G. S. Rohrer, Acta Mater. 51, 3675 (2003).

${ }^{50}$ A. B. Pippard, Response and Stability (Cambridge University Press, Cambridge, 1985), pp. xii+228, an introduction to the physical theory.

${ }^{51}$ M. Ohring, The Materials Science of Thin Films (Academic, San Diego, 1992)

${ }^{52}$ K. Barmak, W. E. Archibald, A. D. Rollett, S. Ta'asan, and D. Kinderlehrer, Grain Boundary Properties and Grain Growth: Al Foils, Al Films, in Interfacial Engineering for Optimized Properties III, edited by Christopher A. Schuh, Mukul Kumar, C. Barry Carter, and Valerie Randle (Mater. Res. Soc. Symp. Proc., Warrendale, PA, 2004), Vol. 819.

${ }^{53}$ R. Courant, K. Friedrichs, and H. Lewy, Math. Ann. 100, 32 (1928).

${ }^{54}$ G. D. Smith, Numerical Solution of Partial Differential Equations, 3rd ed., Oxford Applied Mathematics and Computing Science Series (Clarendon, New York, 1985), pp. xiv+337, finite-difference methods.

${ }^{55}$ Metal Interfaces (American Society for Metals, Cleveland, 1952). 